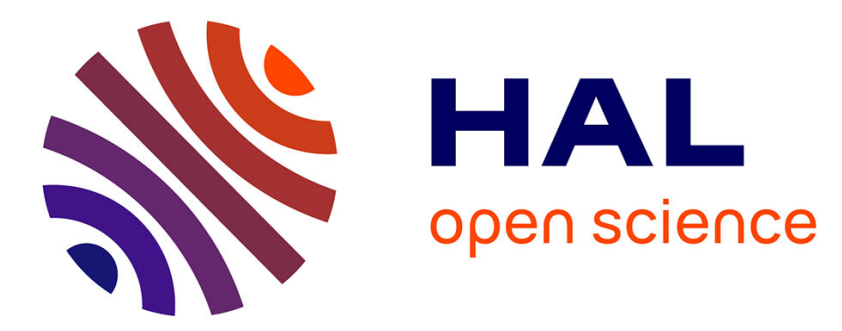

\title{
Columnar phase of pyramidic amphiphiles spread at the air-water interface
}

\author{
A. El Abed, P. Muller, P. Peretti, F. Gallet, J. Billard
}

\section{To cite this version:}

A. El Abed, P. Muller, P. Peretti, F. Gallet, J. Billard. Columnar phase of pyramidic amphiphiles spread at the air-water interface. Journal de Physique II, 1993, 3 (6), pp.851-864. 10.1051/jp2:1993171 . jpa-00247875

\section{HAL Id: jpa-00247875 https://hal.science/jpa-00247875}

Submitted on 1 Jan 1993

HAL is a multi-disciplinary open access archive for the deposit and dissemination of scientific research documents, whether they are published or not. The documents may come from teaching and research institutions in France or abroad, or from public or private research centers.
L'archive ouverte pluridisciplinaire HAL, est destinée au dépôt et à la diffusion de documents scientifiques de niveau recherche, publiés ou non, émanant des établissements d'enseignement et de recherche français ou étrangers, des laboratoires publics ou privés. 


\title{
Columnar phases of pyramidic amphiphiles spread at the air- water interface
}

\author{
A. El Abed ( $\left.{ }^{1}\right)$, P. Muller $\left({ }^{2}\right)$, P. Peretti $\left({ }^{1}\right)$, F. Gallet $\left({ }^{2}\right)$ and J. Billard $\left({ }^{3}\right)$ \\ (1) Laboratoire de Physique et Biophysique, Université René Descartes-Paris V, 45 rue des Saints \\ Pères, 75270 Paris Cedex 06, France \\ (2) Laboratoire de Physique Statistique $\left({ }^{*}\right)$, Ecole Normale Supérieure, 24 rue Lhomond, \\ 75230 Paris Cedex 05, France \\ $\left({ }^{3}\right)$ Laboratoire de Physique de la Matière Condensée (**), Collège de France, Place Marcelin \\ Berthelot, 75232 Paris Cedex 05, France
}

(Received 8 July 1992, revised 28 December 1992, accepted 3 March 1993)

\begin{abstract}
Two compounds, forming thermotropic liquid-crystalline phases in the bulk, were spread at the air-water interface. For both compounds, the surface pressure versus molecular area diagrams exhibit a large domain of molecular areas where the surface pressure of the film is quasiconstant. This plateau region of the isotherms corresponds to a transition from a monolayer in a liquid-expanded phase to a metastable condensed monolayer in which the molecules may adopt an « edge-on » arrangement. In this arrangement, the base of the pyramidic core is normal to the airwater interface. The film was also observed by means of fluorescence and polarizing microscopy. These techniques allowed us to show the formation of anisotropic slowly growing multilayered domains from the « edge-on » monolayer. An original method, based on the light reflectivity of the domains, was developed to measure their thickness and their optical anisotropy. The results show that these domains are formed by an arrangement of the molecules in rectilinear columns for one compound and in spiral columns for the other compound.
\end{abstract}

\section{Introduction.}

Recently, some materials exhibiting thermotropic liquid-crystalline phases in the bulk, were studied at the air water interface. To allow for this kind of study, the compounds should possess an amphitropic behavior: besides their thermotropic behavior, these compounds present an amphiphilic character. Some calamitic [1-4] and discotic [5-9] mesogens were shown to form monolayers at the air-water interface.

(*) Associé au CNRS (URA 1306) et aux Universités Pierre et Marie Curie, Paris VII.

(**) Associé au CNRS (URA 571). 
Using a Langmuir balance, it was shown recently [10] that two pyramidic-like molecules, the hexa-nonanoyl-oxy-tribenzocyclononene and the hexa-nonyloxy-tribenzocyclononene (respectively named ester and ether in the following) can be spread as Langmuir films at the air-water interface. In the bulk, these compounds possess columnar liquid-crystalline phases at a large domain of temperature. The surface pressure-molecular area isotherm diagrams of these films show that during compression, the films undergo transitions that involve not only the aliphatic chains but the whole molecule at the air-water interface. Since three models for the molecular rearrangement could explain these phase transitions, direct observations of the films were necessary.

The visualization of coexisting phases, during a first order phase transition in a Langmuir film, is often made by adding a fluorescent probe to the spreading solutions of films [11]. Despite the addition of impurities (the fluorescent probe) to the film, this technique remains a powerful tool for Langmuir film studies.

In this work and in a first step, a fluorescent dye was added to the spreading solutions of the pyramidic compounds. In a second step, the film was observed directly between a polarizer and an analyzer, without the addition of any dye. At a surface pressure corresponding to the phase transition region and at times which are longer than those necessary for an isotherm run. we observe the formation of anisotropic domains.

In a recent study, Bercegol et al. [12] have observed two dimensional ordered anisotropic domains in monolayers of 12-nitrobenzoxadiazole stearic acid. They measured the reflectivity of these domains, under a normal incident polarized light, to deduce the value of their optical anisotropy. In a more general case, we show in this study that, in addition to the optical anisotropy, one can also measure the thickness of the domains. We apply these results to the films of the pyramidic compounds and show that in the observed domains, the molecules adopt an « edge-on » arrangement, where the pyramidic base of the molecule is perpendicular to the air-water interface, leading to the formation and growth of rectilinear columns for the ester compound and spiral columns for the ether compound.

\section{Experimental.}

The two compounds used for the film preparations were synthetized and purified by Zimmermann et al. [13]. They were discovered in 1985 [13, 14]. The molecules present a pyramidic-like central core to which six long side chains are bound at the pyramid base. These molecules admit in average a three fold symmetry axis (Fig. 1). In the bulk phase, these compounds exhibit mesomorphic phases which are stable at room temperature. Optical microscopy [13] and X-ray patterns $[15,16]$ showed that these mesophases are columnar.

The studied compounds differ by the nature of the function that links the alkyl chain to the pyramidic base :

- hexa-nonanoyloxy-tribenzocyclononene (ester): the long chains are nonanoyl $\left(\mathrm{CH}_{3}\left(\mathrm{CH}_{2}\right)_{7} \mathrm{COO}^{-}\right)$. In the bulk it exhibits a biaxial mesomorphic $\left(P_{\mathrm{D}}\right)$ phase which is stable in the $23.9-152.6^{\circ} \mathrm{C}$ range :

- hexa-nonyloxy-tribenzocyclononene (ether): the long chains are nonyloxy $\left(\mathrm{CH}_{3}\left(\mathrm{CH}_{2,8} \mathrm{O}^{-}\right)\right.$. This compound exhibits a uniaxial mesomorphic $\left(P_{\mathrm{A}}\right)$ 3D phase stable between $18.7^{\circ} \mathrm{C}$ and $66.1{ }^{\circ} \mathrm{C}$.

The high purity of these materials has been checked by TLC, NMR (270 MHz) and combustion analysis [13].

The probe for fluorescence microscopy is a commercial 12-nitrobenzoxadiazole (named NBD) stearic acid (Molecular Probes) and was used without further purification. The NBD group has the particularity of being weakly fluorescent in water. 


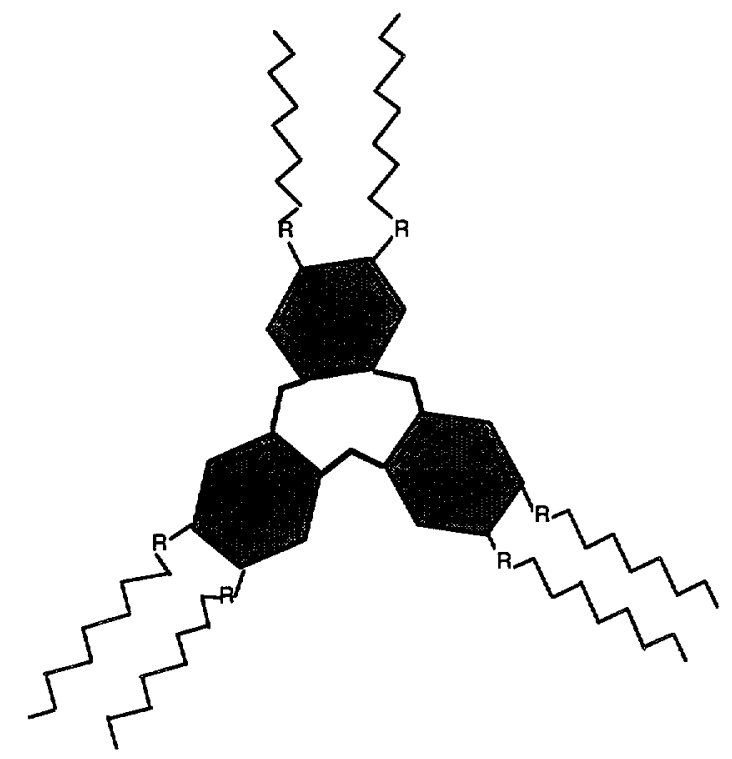

Fig. 1. - Structures of the pyramidic compounds : hexa-nonyloxy-tribenzocyclononene (named ether), the long chains are nonyloxy $\left(\mathrm{R}=\mathrm{CH}_{3}\left(\mathrm{CH}_{2}\right)_{8} \mathrm{O}^{-}\right)$.

The ester and the ether compounds were spread from solutions $2.5 \times 10^{-4} \mathrm{M}$ in chloroform (Normapur from Prolabo). The water was purified in a four cartridge system (ORC-U3-R3-M3 from Bioblock) or in a milliQ system from Millipore.

The diagrams of the surface pressure $(\pi)$ versus the area per molecule $(A)$ were obtained with a film balance (Krüss) at $20^{\circ} \mathrm{C}$. The compression speed was $30 \AA^{2} /($ molecule.mn); the corresponding time for an isotherm run was then about $11 \mathrm{mn}$. The surface pressure was measured with an accuracy of $0.1 \mathrm{mN} / \mathrm{m}$.

The optical observations were made by means of a Polyvar-Met microscope from ReichertJung, set on a home built Langmuir trough. A Hamamatsu video camera with an image intensifier enables us to visualize the film and record images on a video tape. The wavelength of the incident light is about $0.45 \mu \mathrm{m}$. In a first step, fluorescence microscopy experiments have been performed on solutions of the ester and of the ether containing $1 \%$ (by weight) of NBD-stearic acid. In a second step, the amphiphilic film was observed between a polarizer and an analyzer, without the presence of any fluorescent compound.

In order to understand better the nature of the domains, we developed an original method, based on calculation and measurement of the light reflectivity of these domains. This technique was applied, in the absence of the fluorescent probe, to the films of the ester and the ether compounds. The light intensities of the recorded images were measured at the end of the compression (corresponding to a molecular area about $140 \AA^{2} /$ molecule) from the video tape by a Crystal system from Quantel. The comparison of these experimental data to the calculation will allow us to measure the thickness of the domains and their optical anisotropy.

Both observations (thermodynamical and optical) were made at the same temperature and $\mathrm{pH}$.

\section{Results and discussion.}

3.1 THERMODYNAMICAL. - Figure 2 presents the surface pressure $(\pi)$ versus molecular area $(A)$ isotherm diagrams of the pyramidic compounds. For both pyramidic compounds, the 


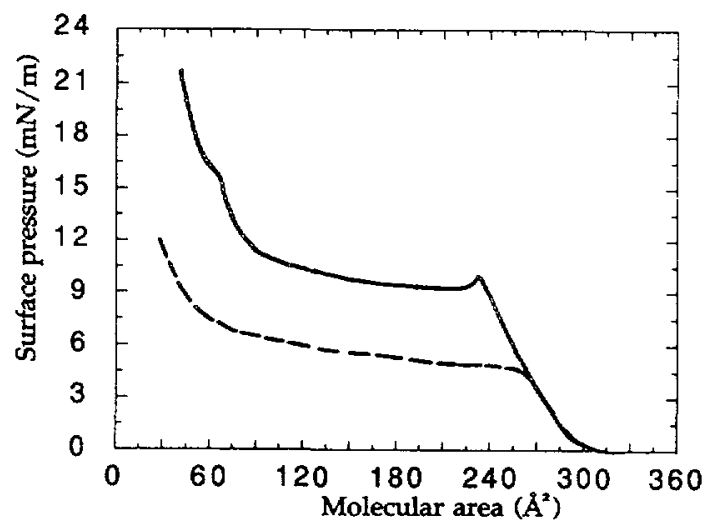

Fig. 2. - Surface pressure $(\pi)$ versus molecular area $(A)$ isotherms; subphase: purified water $\langle\mathrm{pH}=5.7)$, temperature : $20^{\circ} \mathrm{C}$, barrier speed : $30 \AA^{2} /($ molecule.mn), full line : ester, broken line : ether.

surface pressure remains equal to zero, within the accuracy of our measurements, for molecular areas greater than $305 \AA^{2}$. These molecular areas should correspond to a gas-liquid phase transition.

During compression of the ester film, and for molecular areas between 305 and $232 \AA^{2}$, the $\pi-A$ curve increases in a monotonic fashion. This part of the $\pi-A$ isotherm corresponds to a first bidimensional phase. It corresponds to a liquid-expanded (LE) phase. Indeed, the $\pi-A$ isotherms extrapolate to zero $\pi$ at a molecular area about $300 \AA^{2}$ which corresponds to an area about $50 \AA^{2}$ per nonyl chain. This value is in agreement with the molecular area occupied by a single chain of fatty acids in a liquid-expanded monolayer [17]. In this phase, the molecules are «flat» lying at the air-water interface : they should be anchored to the water surface with their six hydrophilic groups. In such a way, the base of their pyramidic core is parallel to the air-water interface while their chains are in a disordered state over the water surface.

The film exhibits a first break in the pressure $\pi$ increase which takes place at $A_{\mathrm{p}_{1}}=232 \AA^{2}$ and which is followed by a small decrease in the surface pressure (hump). The existence of this hump shows that during its compression, the film is not in an equilibrium state. The existence of similar humps is reported in many systems $[2,7,12]$ and it is interpreted as a nucleation barrier for the formation of a new phase (condensed phase). Then a large plateau takes place at $\pi_{p_{1}}$ about $9.5 \mathrm{mN} / \mathrm{m}$. This plateau corresponds to a LE-condensed phase transition. The lowest molecular area value for the condensed phase was observed to be about $A=66 \AA^{2}$ and corresponds to a surface pressure about $16 \mathrm{mN} / \mathrm{m}$.

For the ether compound, the plateau is reached without a hump at $\pi_{\mathrm{p} 2}=4.5 \mathrm{mN} / \mathrm{m}$ (which corresponds to the spreading pressure of the ether) and $A_{\mathrm{p} 2}=264 \AA^{2}$. The pressure increases rapidly for molecular areas below $61 \AA^{2}$. No further transition has been observed for this compound.

If one stops compressing the film from its condensed phase, one observes a relaxation of the surface pressure to that of the plateau transition. This shows that the condensed phase is metastable and hence should lead to a collapse. However, a second compression after a cycle of a compression and a decompression shows identical $\pi-A$ isotherms for the first and the second compression of the film. We conclude that this surface pressure relaxation is due to a reversible collapse. 
The classical liquid expanded-liquid condensed phase transition, usually observed for fatty acid films and which involves solely an ordering of the aliphatic chains, could not explain the low molecular areas of the observed condensed phase. One should consider other kinds of phase transition which involve the whole molecule and not only its chains. Hence, three models (Fig. 3) could explain the low molecular areas of the condensed phase.

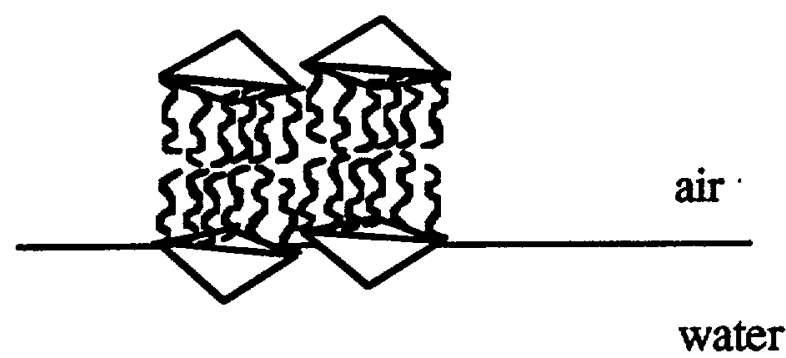

a)

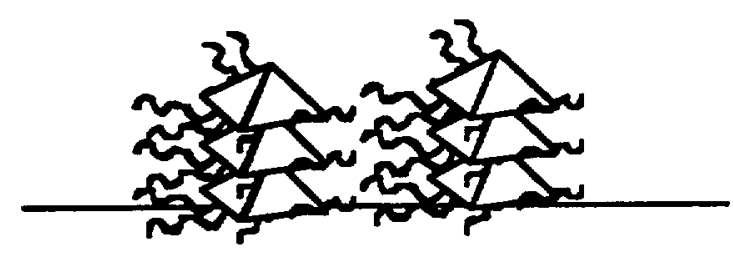

b)

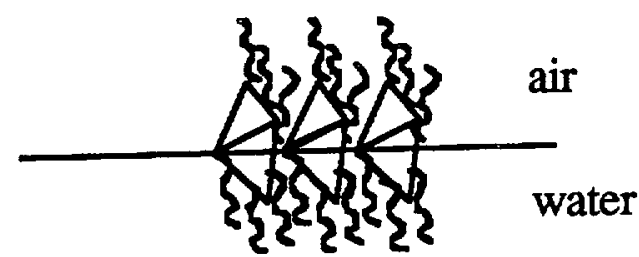

c)

Fig. 3. - Molecular arrangement models of the condensed phase : bilayer in a condensed state (3a) ; expanded 3-layer phase (3b); « edge-on » monolayer (3c).

i) The formation of a bilayer. - In this model (Fig. 3a), the molecules are supposed to be in a condensed state in each layer. The area occupied by one aliphatic chain, in a liquid condensed monolayer, is equal [17] to $22 \AA^{2}$ which means that six chains of each pyramidal molecule would occupy, in a bilayer, an area equals to $\left[\left(6 \times 22 \AA^{2}\right) / 2\right]=66 \AA^{2}$. This value is in agreement with the experimental molecular area at the second transition.

ii) The formation of a 3-layer phase. - Let us suppose that the density of the molecules in each layer of this model (Fig. 3b) is the same as in a the liquid-expanded phase, then the area occupied by each molecule in the three layers is about $77 \AA^{2}:\left(232 \AA^{2} / 3\right)$. This value lies in the range of the molecular areas of the condensed phase. The occurring of such a transition, i.e. a monolayer-3 layer transition, has been shown recently by Kim et al. [18] for the 8-CB (4'-noctyl-4-cyanobiphenyl) liquid crystal Langmuir film. 
iii) An «edge-on" monolayer. - In this model (Fig. 3c), the base of the pyramidic core is no more flat lying at the air-water interface but perpendicular to the air-water interface : in average, three of the aliphatic chains of the molecules are in the water subphase and the three others are in the air. Such arrangements of the molecules lead obviously to a metastable monolayer. It is also in agreement with the observed molecular area at the second transition. The area occupied by three aliphatic chains is indeed $66 \AA^{2}$ if one assumes that the monolayer is in a liquid condensed phase. Albrecht $e t$ al. [7] and Laschewsky [8] have suggested a similar molecular organization for monolayers of discotic liquid crystals bearing short aliphatic chains.

In order to choose between one of these suggested models, we observed the films of the pyramidic compounds by means of a microscope set over the Langmuir trough.

\subsection{OPTICAL.}

3.2.1 Fluorescence. - In fluorescence experiments, and for molecular areas above $305 \AA^{2}$, we have observed for the ether compound, the coexistence of dark bubbles with a brighter phase : a proof for a gas-liquid phase transition. The coexistence of these two phases has been observed with the maximum sensitivity of the camera for the ether but has not been observed for the ester. In the latter case, the non-observation of coexisting phases could be attributed to a poorer contrast between the two phases.

During compression, the microscope field displays a weakly fluorescent homogeneous LE phase. When compressing the film to the plateau areas, small bright domains appear, in the observation field, at the beginning of the LE-condensed phase transition. These domains are narrow elongated stripes of about a few hundred micrometres length and a few micrometres width for the ester compound. For the ether, we observe disc-like islands with a diameter of about a few tens micrometres. When decompressing the monolayer to the LE-phase, these domains disappear for pressures lower than the transition pressure.

The contrast between these domains and the LE phase could be interpreted by assuming that in the liquid phase the probe is anchored in the water subphase by both its acid and NBD groups, while in the bright domains the NBD group is risen up from the subphase. Indeed, the fluorescent group exhibits no fluorescence when it is in an aqueous phase. The quenching of the probe fluorescence in a liquid monolayer is then easily understood. These domains are optically anisotropic in the plane of the air-water interface.

3.2.2 Polarizing microscopy. - The optical anisotropy of both kinds of domains allowed us to visualize their formation even in the absence of the fluorescent probe. Indeed, when a solution of the neat ester or ether is spread on the water subphase and the transition region is reached, the observation between crossed polarizers displays anisotropic domains ( $F_{1}$. 4 and Fig. 5) of similar shape than those observed by fluorescence microscopy. In this case however, the domains appear at time scales which are longer than those required for the observation in the presence of the fluorescent probe. This can be related to the fact that these domains may have a weak optical anisotropy which does not allow their observation below some critical thickness.

It is interesting to note that the main features of the domain shapes remain nearly the same when the observations are made at $10{ }^{\circ} \mathrm{C}, 20^{\circ} \mathrm{C}$ and $26^{\circ} \mathrm{C}$. These results should be related to those reported by many authors $[20,21]$ about the influence of the nature of a surface on the surface phase transitions.

Ester compound. - The brighter domains have their long axes oriented at $\pm 45^{\circ}$ with respect to the electric field of the incident polarized light. The domains whose long axes are parallel or perpendicular to the polarizing axis are not seen. This shows that the neutral lines are parallel and perpendicular to the long axes of the domains. 

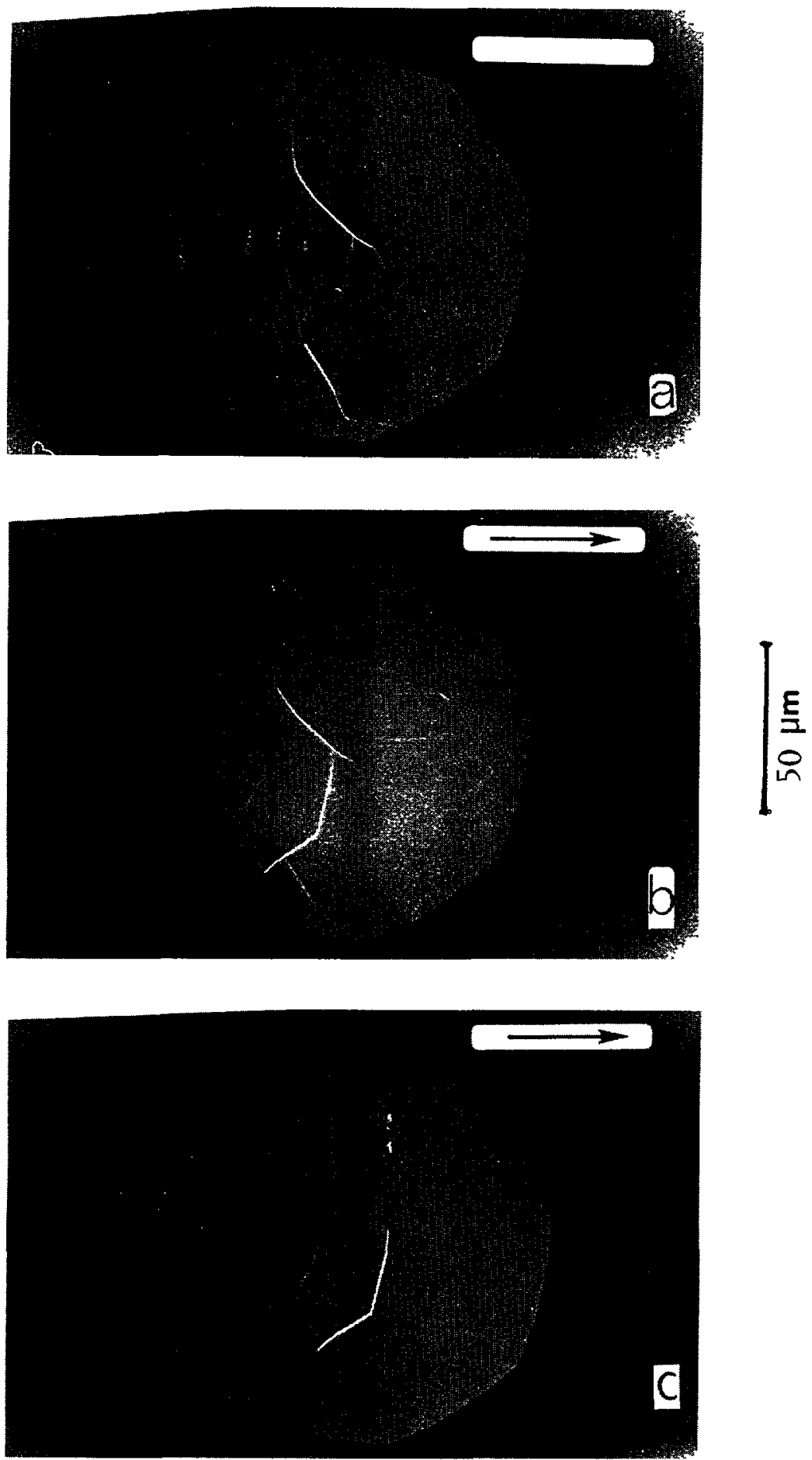

Fig. 4. - Anisotropic domains observed in the ester film between a polarizer and an analyzer; molecular area : $140 \AA^{2}$, temperature : $20^{\circ} \mathrm{C}$, subphase : purified water $(\mathrm{pH}=5.7)$; (a) : the analyzer makes an angle $\Delta \theta=5$ degrees with the polarizer; (b): $\Delta \theta=0$ degrees; (c) : $\Delta \theta=-5$ degrees. The arrows indicate the direction of the vibration transmitted by the polarizer. 

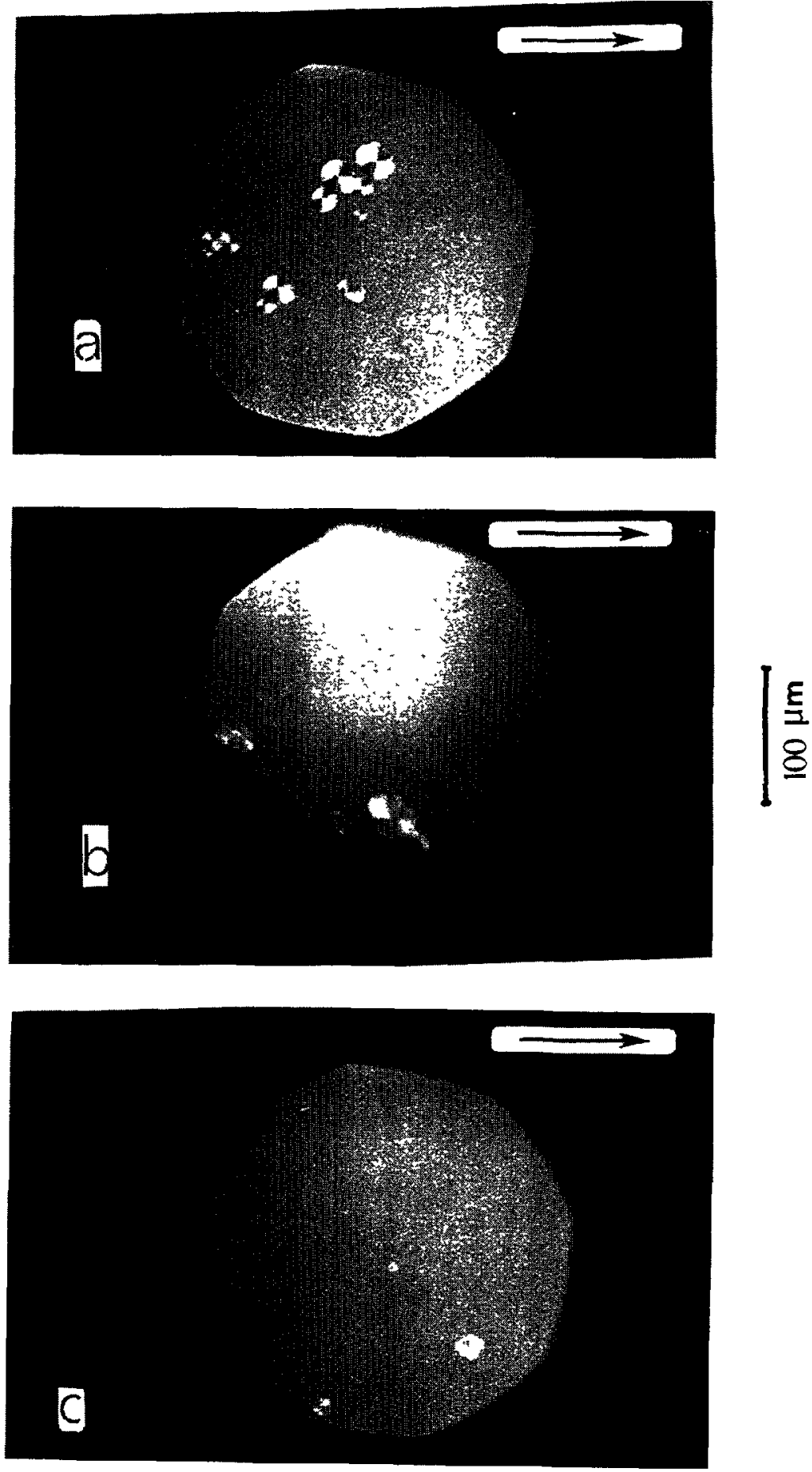

Fig. 5. - Anisotropic domains observed in the ether film between a polarizer and an analyzer : molecular area : $140 \AA^{2}$, temperature : $20^{\circ} \mathrm{C}$, subphase : purified water $(\mathrm{pH}=5.7)$; (a) : the analyzer makes an angle $\Delta \theta=5$ degrees with the polarizer: (b) : $\Delta \theta=0$ degrees; (c) : $\Delta \theta=-5$ degrees. The arrows indicate the direction of the vibration transmitted by the polarizer. 
We define the contrast as the ratio of the reflected light intensity of an anisotropic domain over the reflected light intensity of the background. When the analyzer is rotated by an angle $\Delta \theta$ (a few degrees) with respect to the initial crossed position of the analyzer, the contrast of the domains oriented at $-45^{\circ}$ increases when $\Delta \theta$ is negative and decreases when $\Delta \theta$ is positive. On the contrary, for those domains whose long axes are oriented at $+45^{\circ}$ with regard to the polarizing direction the contrast increases when $\Delta \theta$ is positive and decreases when $\Delta \theta$ is negative.

Ether compound. - The typical domains display bright quadrants which are all visible when the polarizer and the analyzer are crossed. These quadrants are separated by sharp dark lines which are parallel and perpendicular to the polarizer. The contrast of the first and the third quadrants increases when $\Delta \theta$ is negative and decreases when $\Delta \theta$ is positive. For the other two quadrants, the contrast increases when $\Delta \theta$ is positive and decreases when $\Delta \theta$ is negative. The neutral lines of the observed domains are radial and tangential.

3.2.3 Reflectivity of the anisotropic domains. - We calculate, under a normal incidence, the intensity of the reflected light as a function of the angle $\Delta \theta$ (see appendix).

Experimentally, we recorded images of the domains at different values of $\Delta \theta$, then we measured the light intensities of the isotropic background $I_{\mathrm{b}}$ and of the anisotropic domain $I_{\mathrm{A}}$, along the two directions $+45^{\circ}$ and $-45^{\circ}$. The $I_{\mathrm{A}}$ and $I_{\mathrm{b}}$ values were measured by the image analyzer in relative units.

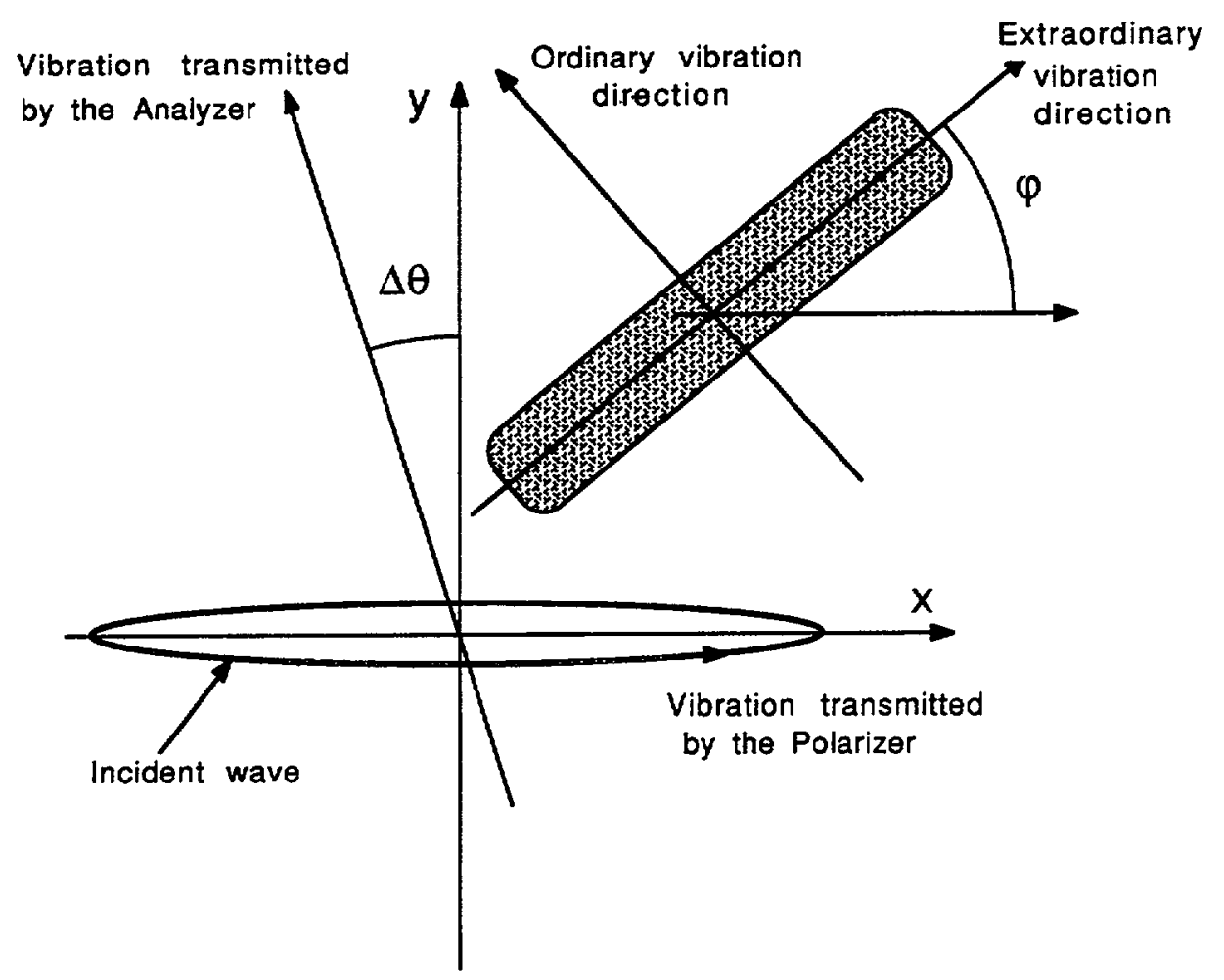

Fig. 6. - An anisotropic domain is normally illuminated by an incident polarized light, with small ellipticity $\delta$ along the $O x$ axis. The extraordinary axis makes an angle $\varphi$ with the $O x$ axis. The domain is then observed through an analyzer that makes an angle $\pi / 2+\Delta \theta$ with the $\mathrm{O} x$ axis. 
The experimental curves $I_{\mathrm{A}}$ and $I_{\mathrm{b}}$ versus $\Delta \theta$ are parabolic (Fig. 7) and this is in agreement with the relation (1) given in the appendix. A non-linear fit of these curves gives the values of the parabola coefficients. The coefficient $\beta$ is directly proportional to the thickness $d$ of the domain. The quantities $\left(A_{\mathrm{e}}-A_{0}\right)$ and $\left(C_{\mathrm{e}}-C_{0}\right)$ are proportional to the optical anisotropy which is defined as $\Delta n=n_{\mathrm{e}}-n_{0}$ where $n_{\mathrm{e}}$ and $n_{0}$ are respectively the extraordinary and the ordinary indexes. The mean refractive index $n$ of both compounds has been measured in the bulk by refractometry. We found:

$$
n(\text { ester })=1.523 \text { and } n \text { (ether) }=1.512 \text {. }
$$

Thus the parameters of the fit are : $\Delta n, d$ and $\delta$.

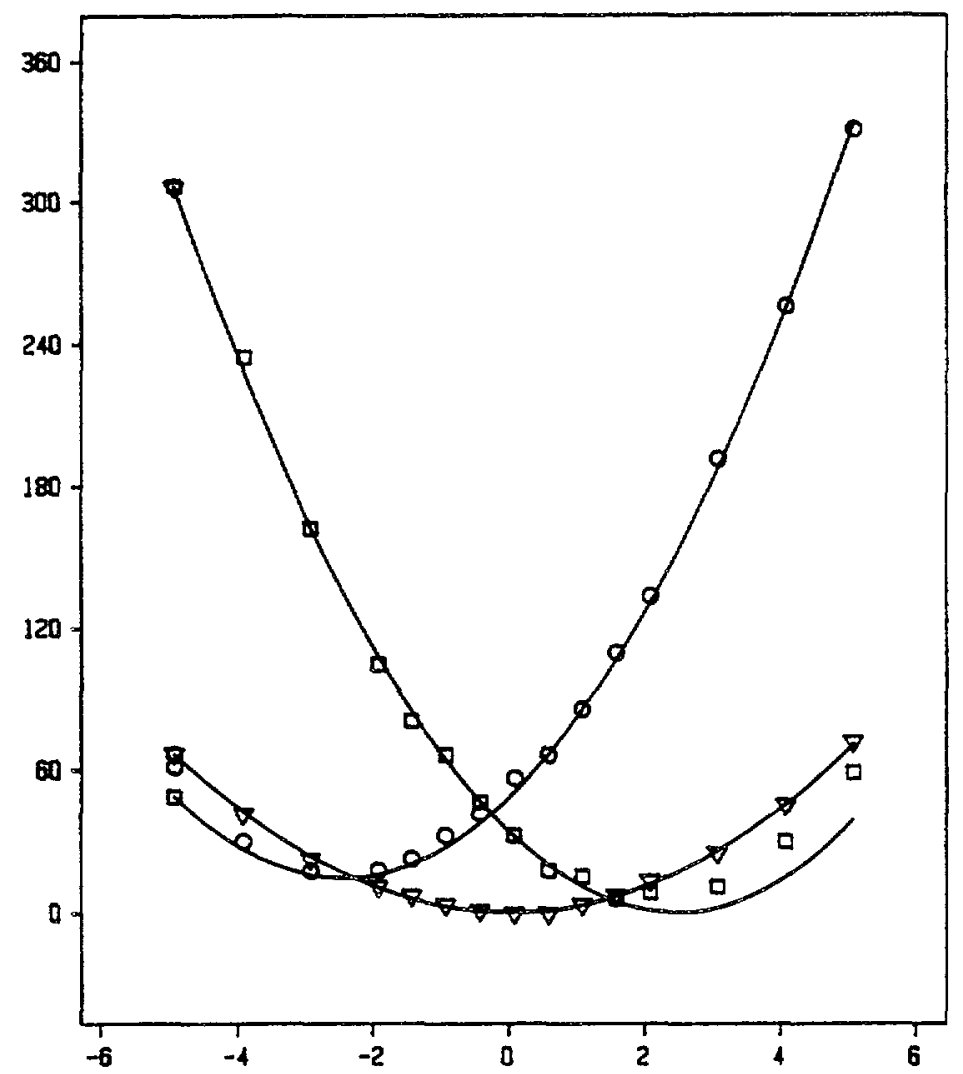

Fig. 7. - Experimental reflected intensity $I_{\mathrm{A}}$ versus $\Delta \theta$ for a circular domain of the ether compound. First and third quadrants $(\varphi=+45$ degrees $):(0)$; second and fourth quadrants $(\varphi=-45$ degrees $)$ : $(\square)$. Experimental reflected intensity $I_{\mathrm{h}}$ versus $\Delta \theta$ for the background $:(\nabla)$. The tull line curves are the best theoretical fits. The thickness $d$ and the optical anisotropy of this domain was found to be equal to $d=302 \AA, \Delta n=n_{\mathrm{e}}-n_{0}=-0.04$.

The analysis of the curves $I_{\mathrm{A}}=f(\Delta \theta)$ from different experiments, at constant molecular area (140 $\AA^{2} /$ molecule) and at different time scales, shows that the measured thicknesses vary from 60 to $230 \AA$ for the ester and from 116 to $325 \AA$ for the ether. These thicknesses show that these domains are multilayered. Indeed, a maximum value of about $20 \AA$ for the monolayer 
thickness can be deduced from X-ray data [15-16] in the bulk. It appears that this method is not sensitive enough to detect a monolayered domain. It is important to note that the formation dynamics of these 3D domains were observed to be slow. The thinnest domains were obtained about half an hour after the plateau has been reached and the thickest ones were obtained two days later.

Another result, deduced from the analysis of the curves $I_{\mathrm{A}}=f(\Delta \theta)$, is the calculation of the optical anisotropy $\Delta n$ of these domains. It was found to be about -0.05 for the ester compound and -0.04 for the ether compound. Let us notice that $\Delta n$ is negative, as usually observed for columnar phases. We were not able yet to compare these optical anisotropies to those of the studied mesophases in the bulk. Indeed, these compounds display in the bulk a nonhomogeneous orientation which inhibits any measurement of the ordinary or of the extraordinary refractive indexes.

The small ellipticity $\delta$ of the incident light was observed to depend upon the position in the microscope field and varies from a few tenths of a degree to few degrees. This confirms that the ellipticity may originate from a residual birefringence of the objective microscope.

To explain qualitatively the optical anisotropy, in the plane of the interface, of the domains, one has to admit that the molecules are stacked in columnar arrays (Fig. 8) : the stacking is rectilinear for the ester and rather spiral (or concentric) around the central defect point for the ether. This means that the molecules are « edge-on » in the domains.

(a)
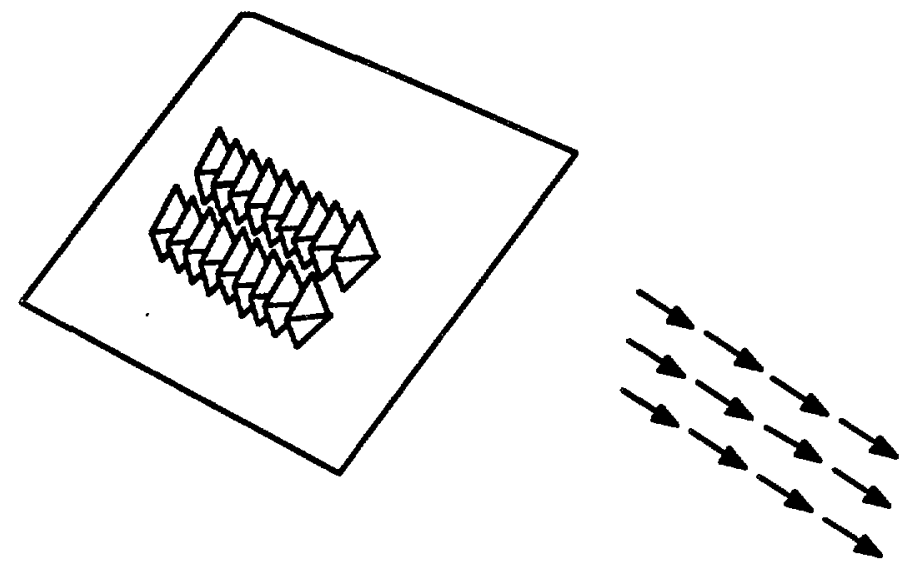

(b)
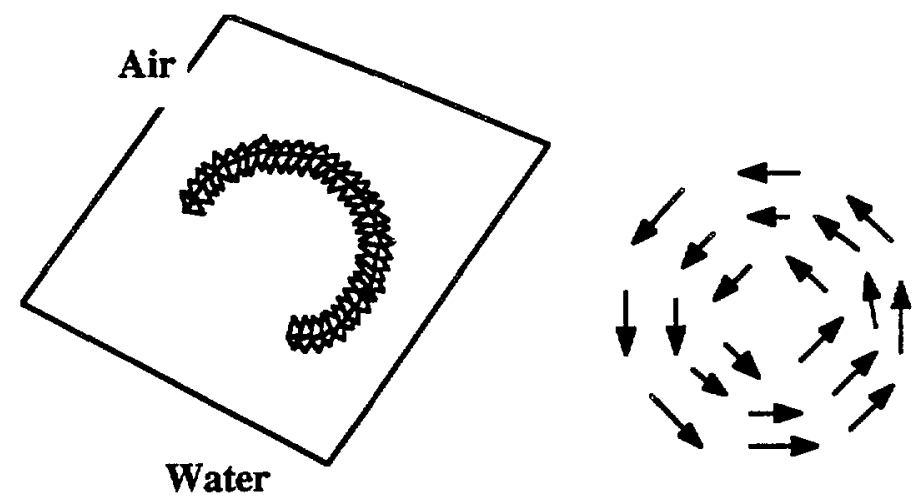

Fig. 8. - Molecular arrangement models in the 3D domains : a) rectilignar columns for domains of ester compound; b) concentric columns for domains of the ether compound. The arrows indicate the extraordinary neutral line. 
It is interesting to note that the molecules, in the bulk, also associate in columnar mesophases and that the observed domains in the film are very similar to the bulk growth shapes, observed at the transition from the isotropic liquid to the liquid-crystalline phase for both compounds.

As was mentioned below, the observation of the film in the presence of the fluorescent probe allows us to visualize the formation of anisotropic domains at the beginning of the transition. At this stage, the domains are probably still bidimensional. Their optical anisotropy in the plane of the air-water interface shows that they also possess an "edge-on " structure. The determination of such a structure for both tridimensional and bidimensional domains indicates that the molecules may also adopt during compression, an «edge-on » arrangement in the metastable condensed monolayer. At longer times scales and at constant molecular area, this metastable monolayer is slowly transformed in to 3D domains.

\section{Conclusion.}

We have shown that two pyramidic compounds can form monolayers at the air-water interface. During compression, the surface pressure versus molecular area isotherms exihibit the existence of two phases : a liquid-expanded phase and a condensed phase. At large molecular areas, the film is in the liquid-expanded state. This stable phase is formed by a flat arrangement of the pyramidic base of the molecules at the air-water interface. The transition from the expanded to the condensed phase is characterized by a large quasi-plateau. At molecular areas corresponding to the condensed phase, the molecules may adopt an edge-on arrangement and form a metastable monolayer which undergoes the formation of a $3 \mathrm{D}$ phase. The visualization of the film at the phase transition region has shown the formation of anisotropic domains in the plane of the air-water interface. An original method, based on the reflectivity measurements of these multilayered domains, has been developed to deduce their thickness values and their optical anisotropy.

\section{Appendix.}

We calculate here the intensity of the reflected light by an anisotropic domain of the ester compound illuminated with a linearly polarized light. The domains are very thin and their thickness $d$ is small in comparison with the incident wavelength $\lambda$. We assume that the optical axis lies in the plane of the ester domains and that the extraordinary and the ordinary axes are respectively parallel and perpendicular to the long axes of the domains. Then $n_{\mathrm{e}}$ and $n_{0}$ are respectively the extraordinary and the ordinary refractive indexes.

The phase and the amplitude of the electric field of the reflected light $E_{\mathrm{e}}$, along the extraordinary axis depend upon the amplitude $E_{\mathrm{i}}$ of the incident field and the angle $\varphi$ between $E_{\mathrm{i}}$ and this axis (Fig. 6). Let $r_{\mathrm{ae}}$ and $r_{\text {we }}$ be respectively the reflectivity coefficients for the air-film and the film-water interfaces along the extraordinary axis, then one obtains in the complex representation :

$$
\begin{gathered}
E_{\mathrm{e}}=R_{\mathrm{e}} E_{\mathrm{l}} \cos \varphi \quad \text { with } \quad R_{\mathrm{e}}=\frac{r_{\mathrm{ae}}+r_{\mathrm{we}} \mathrm{e}^{j_{\mathrm{e}} \beta}}{1+r_{\mathrm{ae}} r_{\mathrm{we}} \mathrm{e}^{\mathrm{e}_{\mathrm{e}} \beta}} \\
\beta=4 \pi \frac{d}{\lambda}, \quad r_{\mathrm{ae}}=\frac{n_{\mathrm{e}}-n_{\mathrm{a}}}{n_{\mathrm{e}}+n_{\mathrm{a}}}, \quad r_{\mathrm{we}}=\frac{n_{\mathrm{e}}-n_{\mathrm{w}}}{n_{\mathrm{e}}+n_{\mathrm{w}}}
\end{gathered}
$$

where $n_{\mathrm{w}}, n_{\mathrm{a}}$ are the refractive indexes for the water and the air, respectively, and $\lambda$ the wavelength of the incident light. Taking the small thickness of the domains into account, 
$R_{\mathrm{e}}$ can be expressed, to a second order in $\beta$, as follows :

$$
R_{\mathrm{e}}=R_{\mathrm{aw}}\left(1+j \beta A_{\mathrm{e}}+\beta^{2} B_{\mathrm{e}}\right)
$$

where $\quad R_{\mathrm{aw}}=\frac{n_{\mathrm{w}}-n_{\mathrm{a}}}{n_{\mathrm{w}}+n_{\mathrm{a}}}, \quad A_{\mathrm{e}}=\frac{n_{\mathrm{a}}\left(n_{\mathrm{w}}^{2}-n_{\mathrm{e}}^{2}\right)}{n_{\mathrm{a}}^{2}-n_{\mathrm{w}}^{2}}, \quad B_{\mathrm{e}}=\frac{n_{\mathrm{a}}\left(n_{\mathrm{e}}^{2}-n_{\mathrm{w}}^{2}\right)\left(n_{\mathrm{e}}^{2}+n_{\mathrm{w}} n_{\mathrm{a}}\right)}{2\left(n_{\mathrm{w}}^{2}-n_{\mathrm{a}}^{2}\right)\left(n_{\mathrm{w}}+n_{\mathrm{a}}\right)}$

Similar expressions can be obtained for the component $E_{0}$ of the reflected light along the ordinary axis by replacing the index «e » by the index $« 0 »$ and $\varphi$ by $(\varphi+\pi / 2)$.

Because of the residual birefringence of the microscope objectives, we have to take into account a small ellipticity $\delta$ of the incident light. The reflected field component $E_{\mathrm{A}}$ along the analyzer, which makes an angle $(\pi / 2+\Delta \theta)$ with the polarizer direction, can be then expressed by :

$E_{\mathrm{A}}=E_{\mathrm{e}} \sin (\varphi-\Delta \theta)-E_{0} \cos (\varphi-\Delta \theta)$

$E_{\mathrm{A}}=E_{1}\left[R_{\mathrm{e}}(\cos \delta \cos \varphi+j \sin \delta \sin \varphi) \sin (\varphi-\Delta \theta)-\right.$

$\left.-R_{0}(\cos \delta \sin \varphi-j \sin \delta \cos \varphi) \cos (\varphi-\Delta \theta)\right]$

Therefore the intensity $I_{\mathrm{A}}$ of the reflected light on the domain after the analyzer is :

$$
I_{\mathrm{A}}=I_{0}\left(K_{1} \sin ^{2} \Delta \theta+K_{2} \cos ^{2} \Delta \theta+K_{3} \cos \Delta \theta \sin \Delta \theta\right),
$$

where $I_{0}=\left(R_{\mathrm{aw}}\right)^{2}\left(E_{\mathrm{j}}\right)^{2}$ is the reflected light intensity in the absence of the Langmuir film. The coefficients $K_{1}, K_{2}$ and $K_{3}$ are given by :

$$
\begin{gathered}
K_{1}=\left[1+2\left(B_{\mathrm{e}} \cos ^{2} \varphi+B_{0} \sin ^{2} \varphi\right) \beta^{2}+\left(A_{\mathrm{e}} \cos ^{2} \varphi+A_{0} \sin ^{2} \varphi\right)^{2} \beta^{2}\right] \cos ^{2} \delta+ \\
+\cos ^{2} \varphi \sin ^{2} \varphi\left(A_{\mathrm{e}}-A_{0}\right)^{2} \beta^{2} \sin ^{2} \delta-2 \beta\left(A_{\mathrm{e}}-A_{0}\right) \cos \varphi \sin \varphi \cos \delta \sin \delta \\
K_{2}=\left[1+2\left(B_{\mathrm{e}} \sin ^{2} \varphi+B_{0} \cos ^{2} \varphi\right) \beta^{2}+\left(A_{\mathrm{e}} \sin ^{2} \varphi+A_{0} \cos ^{2} \varphi\right)^{2} \beta^{2}\right] \sin ^{2} \delta+ \\
+\cos ^{2} \varphi \sin ^{2} \varphi\left(A_{\mathrm{e}}-A_{0}\right)^{2} \beta^{2} \cos ^{2} \delta+2 \beta\left(A_{\mathrm{e}}-A_{0}\right) \cos \varphi \sin \varphi \cos \delta \sin \delta \\
K_{3}=2 \beta^{2}\left[B_{0}-B_{\mathrm{e}}+\left(A_{0}-A_{\mathrm{e}}\right)\left(A_{\mathrm{e}} \cos ^{2} \varphi+A_{0} \sin ^{2} \varphi\right)\right] \cos \varphi \sin \varphi \cos ^{2} \delta+ \\
+2 \beta^{2}\left[B_{0}-B_{\mathrm{e}}+\left(A_{0}-A_{\mathrm{e}}\right)\left(A_{\mathrm{e}} \sin ^{2} \varphi+A_{0} \cos ^{2} \varphi\right)\right] \cos \varphi \sin \varphi \sin ^{2} \delta+ \\
+2 \beta\left(A_{\mathrm{e}}-A_{0}\right) \times\left(\sin ^{2} \varphi-\cos ^{2} \varphi\right) \cos \delta \sin \delta .
\end{gathered}
$$

The brighter domains are those for which $\varphi=\varepsilon 45^{\circ}$, where $\varepsilon= \pm 1$.

As $\Delta \theta$ and $\delta$ are small, one can simplify the above relation into:

$$
\frac{I_{\mathrm{A}}}{I_{0}}=\Delta \theta^{2}\left[1+\left(B_{\mathrm{e}}+B_{0}+A_{\mathrm{e}} A_{0}\right) \beta^{2}\right]+\varepsilon \Delta \theta\left(C_{0}-C_{\mathrm{e}}\right) \beta^{2}+K
$$

where

$$
\begin{gathered}
K=\frac{\beta^{2}}{4}\left(A_{\mathrm{e}}-A_{0}\right)^{2}+\varepsilon \beta \delta\left(A_{\mathrm{e}}-A_{0}\right)+\delta^{2}\left[1+\beta^{2}\left(B_{\mathrm{e}}+B_{0}\right)\right]^{2}, \\
C_{\mathrm{e}}=B_{\mathrm{e}}+\frac{A_{\mathrm{e}}^{2}}{2} \quad \text { and } \quad C_{0}=B_{0}+\frac{A_{0}^{2}}{2}
\end{gathered}
$$

The relation (1) shows that the reflected light intensity $I_{\mathrm{b}}$ of the isotropic background is obtained when $A_{\mathrm{e}}=A_{0}$ and $C_{\mathrm{e}}=C_{0}$. The background corresponds to the film region where there are no anisotropic structures. The curve $I_{\mathrm{b}}$ versus $\Delta \theta$ is a parabola which is symmetric with regard to the intensity axis. Similar results can be established for the ether. 


\section{Acknowledgments.}

The authors wish to acknowledge the contribution of Prof. M. Warenghem (USTL-Lille, France) for the measurement of the mean refractive index. The pyramidic compounds were supplied by H. Zimmermann (Max Planck Institut-Heidelberg, Germany) to whom we are thankful.

\section{References}

[1] Dorfler H. D., Kercher W. and Sackmann H., Z. Phys. Chem. (Leipzig) 251 (1972) 314.

[2] Diep-Quang X. and Uberreiter K., Colloid Polymer Sci. 258 (1980) 1055.

[3] Rapp B. and Gruler H., Phys. Rev. A 42 (1990) 2215.

[4] Rapp B., Eberhardt M. and Gruler H., Makromol. Chem. 46 (1991) 439.

[5] Rondelez F. and Koppel D., J. Phys. France 43 (1982) 1371.

[6] Rondelez F., Baret J. F. and BoIs A. G., J. Phys. France 48 (1987) 1225.

[7] Albrecht O., Cumming W., Kreuder W., Laschewsky A. and Ringsdorf H., Colloid Polymer Sci. 264 (1986) 659.

[8] Laschewsky A., Angew. Chem. Adv. Mater. 101 (1991) 347.

[9] Maliszewsky N. C., Heiney P. A., Blasie J. K., McCauley J. P. and Smith III A. B., J. Phys. II France 2 (1992) 75.

[10] El Abed A., Hochapfel A., Hasmonay H., Billard J., Zimmermann H., Luz Z. and Peretti P., Thin Solid Films 210 (1992) 93.

[11] Losche M. and Mohwald H., Rev. Sci. Inst. 55 (1984) 1968.

[12] Bercegol H., Gallet F., Langevin D. and Meunier J., $J$. Phys. France 50 (1989) 2277.

[13] Zimmermann H., Poupko R., Luz Z. and Billard J., Z. Naturforsch. 40a (1985) 149.

[14] Malthete J. and Collet A., Nouv. J. Chimie 9 (1985) 151.

[15] Levelut A. M., Malthete J. and Collet A., J. Phys. France 47 (1986) 351.

[16] Poupko R., Luz Z., Spielberg N. and Zimmermann H., J. Am. Chem. Soc. 111 (1989) 6094.

[17] Adamson A. W.. Physical Chemistry of Surface (Interscience Publishers, 2nd edn. New York, 1967) pp. 141-144.

[18] Xue J., Jung C. S. and KIM M. W., Phys, Rev. Lett. 69 (1992) 474.

[19] Miyano K., Phys. Rev. Lett. 43 (1979) 51.

[20] Pershan P. S. and Als-Nielsen J., Phys. Rev. Lett. 52 (1984) 759.

[21] Ocko B. M., Braslau A., Pershan P. S., Als-Nielsen J. and Deutsch M., Phys. Rev. Lett. 57 (1986) 94. 